\title{
GRAVIDEZ I MATERNIDADE E ADOLESCENTES EM SITUAÇÃO DE RISCO SOCIAL E PESSOAL: ALGUMAS CONSIDERAÇÕES
}

\author{
PREGNANCY / MATERNITY AND ADOLESCENTS IN SOCIAL AND/ PERSONAL RISK SITUATION: SOME \\ CONSIDERATIONS ABOUT THIS THEME
}

\begin{abstract}
EMBARAZO / MATERNIDAD Y ADOLESCENTES EN SITUACIÓN DE RIESGO SOCIAL Y PERSONAL: ALGUNAS CONSIDERACIONES
\end{abstract}

\author{
Daniela Tavares Gontijo ${ }^{1}$ \\ Marcelo Medeiros ${ }^{2}$
}

\begin{abstract}
RESUMO: A gravidez/maternidade na adolescência é amplamente abordada na literatura, no entanto é possível observar as contradições nos discursos que caracterizam esta experiência como um problema de saúde pública e relatos das adolescentes. Este artigo, caracterizado como um estudo bibliográfico objetivou discutir as diferentes percepções sobre a maternidade na adolescência, entendida como fenômeno socialmente constituído. Estudos que caracterizam a gravidez /maternidade como sendo de risco para o desenvolvimento pessoal e social da adolescente e seu filho, foram contrapostos às pesquisas que trazem a perspectiva da adolescente sobre esta vivência. Constatou-se que a maternidade pode ser vista de forma positiva pela adolescente, especialmente, para aquelas em situação de risco social e pessoal. Assim sendo, observou-se a necessidade de novas pesquisas que abordem a vivência da maternidade através de um eixo sócio-cultural, objetivando em ultima instância a elaboração de políticas públicas efetivas direcionadas a essa população.
\end{abstract}

PALAVRAS CHAVES: Maternidade; Gravidez; Adolescência.

ABSTRACT: The pregnancy and maternity in the adolescence is an widely boarded subject in literature, however is possible to observe the contradictions in the speeches that characterize this experience as a problem of public health and stories of the adolescents on the daily one while mothers. This article, characterized as a bibliographical study objectified to argue the different perceptions on the maternity in the adolescence, understood as phenomenon socially consisting. Studies that characterize the pregnancy/maternity as being of risk for the personal and social development of the adolescent and its son, had been opposed to the research that brings the perspective of the adolescent on this experience. It was possible to evidence that the maternity can be seen of positive form for the adolescent, and especially, for those in situation of social and personal risk, it can represent a rich period of changes as for daily practical the social recognition and to its, besides being an especially propitious moment for the action development health effective.

KEYWORDS: Maternity; Pregnancy; Adolescence.

RESUMEN: El embarazo/maternidad en la adolescencia es ampliamente abordado en la literatura, sin embargo es posible observar las contradicciones en los discursos que caracterizan esta experiencia como un problema de salud pública y relatos de las adolescentes. Este artículo caracterizado como un estudio bibliográfico ha objetivado discutir las diferentes percepciones sobre la maternidad en la adolescencia, entendida como fenómeno socialmente constituido. Estudios que caracterizan el embarazo/maternidad como siendo de riesgo. Para el desenvolvimiento personal y social de la adolescente y su hijo, han sido contrapuestos a las pesquisas que traen la perspectiva de la adolescente sobre esta vivencia. Se ha constatado que la maternidad puede ser vista de forma positiva por la adolescente, especialmente, para aquellas en situación de riesgo social y personal. Siendo así, se ha observado la necesidad de nuevas pesquisas que aborden la vivencia de la maternidad a través de un eje socio-cultural, objetivando en ultima instancia la elaboración de políticas públicas efectivas direccionadas a esa población.

PALABRAS CLAVES: Maternidad; Embarazo; Adolescentes.

\footnotetext{
1 Terapeuta Ocupacional. Mestranda em Ciências da Saúde: Programa Interinstitucional de Pós Graduação - Mestrado e Doutorado Unb/UFG/UFMS; Goiânia (GO) danigontijo@hotmail.com

2 Enfermeiro. Doutor em Enfermagem. Professor Adjunto da Faculdade de Enfermagem da Universidade Federal de Goiás. marcelo@fen.ufg.br
} 


\section{INTRODUÇÃO}

A situação de desigualdade social, política e econômica encontrada no Brasil tem influência direta na dinâmica familiar e no aumento do número de crianças e adolescentes em situação de risco social e pessoal. Nesse contexto, a gravidez na adolescência tem sido tradicionalmente tratada como um problema de saúde pública, apesar de diferentes estudos atribuírem significações positivas para a vivência da maternidade a partir da perspectiva das adolescentes.

O presente artigo tem como proposta discutir as diferentes significações dadas à gravidez/maternidade na adolescência, a partir de artigos científicos publicados sobre o tema. Para sua sistematização, inicialmente serão abordados aspectos relacionados à metodologia utilizada e à caracterização de crianças e adolescentes em situação de risco social e pessoal. Portanto, trata-se de um artigo revisão, elaborado a partir de bibliografia catalogada na base de dados Lilacs (consulta em maio de 2004). Na busca utilizamos descritores como gravidez, maternidade, adolescência e risco social e pessoal. A partir daí selecionamos os artigos que abordassem a gravidez ou maternidade na adolescência a partir de um eixo sócio-cultural. Esta escolha justifica-se pela necessidade de entendermos a gravidez/maternidade enquanto fenômeno socialmente constituído e significado de acordo com o contexto sócio cultural no qual se insere. Assim, traçamos dois eixos de discussão a partir do que encontramos nos textos, isto é, a situação de risco pessoal e social pra o desenvolvimento da adolescente e de seu filho e, a possibilidade de novas formas de relacionamentos e reconhecimentos sociais e de atuação no cotidiano.

\section{A CRIANÇA E ADOLESCENTE EM SITUAÇÃO DE RISCO SOCIAL E PESSOAL:}

Após 14 anos da publicação do Estatuto da Criança e do Adolescente - ECA (BRASIL, 1990), observa-se que se por um lado, houveram ganhos no que se refere à legislação protetiva, por outro a sua implementação tem sido marcada por dificuldades políticas, de disponibilização de recursos humanos e financeiros e de gerenciamento (GALHEIGO et al, 2001; RIZZINI \& SILVA, 2002; SARTI, 1995).

De acordo com dados do IBGE, citados por RIZZINI \& SILVA (2002), em 1999 no Brasil, 39\% das crianças de 0 a 6 anos viviam em famílias com rendimento mensal de até meio salário mínimo, sendo esta percentagem de $37,2 \%$ e $20,2 \%$ para as faixas etárias de 7 a 14 anos e 15 e 17, respectivamente. Estes dados ilustram a situação de desigualdade social, política e econômica que influencia diretamente na dinâmica familiar e na escolha da rua como forma de sobrevivência para um número cada vez maior de crianças e adolescentes (MEDEIROS \& FERRIANI, 1995; RIZZINI \& SILVA, 2002; MEDEIROS et al, 2002; CARVALHO \& GUARÁ, 1994).

Nesse sentido, CARVALHO \& GUARÁ (1994) afirmam que "atrás de crianças e adolescentes em abandono existem famílias abandonadas, esquecidas pelos programas das diferentes políticas sociais $\mathrm{e}$ negligenciadas até mesmo pela política de assistência social“" (p. 46).

A escolha pela rua como opção de sobrevivência, não significa necessariamente, a dissolução dos laços familiares (CARVALHO \& GUARÁ, 1994; MELLO, 1994; MEDEIROS, 2002). No entanto observam-se crianças e adolescentes que vivem e moram pelas ruas, mantendo ou não o vínculo familiar. Nesse sentido, concordamos com MEDEIROS (1995) que, utiliza a denominação "crianças e adolescentes em situação de rua" como caracterização da utilização deste espaço "como uma estratégia de garantia de sua sobrevivência e/ou de familiares, ainda que expostas aos riscos sociais e pessoais característicos da rua".

Estudos estatísticos, citados por MEDEIROS et al (2002) apontam que o perfil de crianças e adolescentes que vivem nas ruas, no Brasil, é caracterizado por baixo nível de escolaridade, cor negra, idade entre 07 e 17 anos. Em Goiânia, o maior contingente encontra-se na faixa de 11 a 14 anos. MEDEIROS et al (2002) apontam que a violência permeia toda a trajetória de vida dessas crianças e adolescentes, impossibilitando condições mínimas de qualidade de vida e diminuindo drasticamente as possibilidades destas se desenvolver plenamente.

Com base nestes aspectos entendemos por situação de risco , concordando com Lescher (2004, p. 11) , como a condição que crianças e adolescentes que " por suas circunstâncias de vida, estão expostas à violência, ao uso de drogas e a um conjunto de experiências relacionadas à privações de ordem afetiva, cultural e socioeconômica que desfavorecem o pleno desenvolvimento bio-psico-social" . Neste sentido, podemos entender os riscos sociais e pessoais como" "possíveis fatores que podem prejudicar $\mathrm{o}$ processo de crescimento e desenvolvimento ou mesmo limitar sua qualidade de vida" (MEDEIROS, 1999, p. 48).

\section{ADOLESCÊNCIA DE RISCO E O RISCO DA GRAVIDEZ}

\section{A adolescência de risco social:}

Embora a adolescência seja um assunto amplamente abordado na literatura científica, não se encontra um consenso sobre a definição da faixa etária na qual se enquadra. Essas controvérsias se justificam pelo caráter multifacetado da adolescência, sendo esta definida de acordo com as especificidades dos pesquisadores. Segundo COSTA (2000) a Organização Mundial de Saúde define a juventude entre 15 e 25 anos, sendo que a adolescência corresponderia à faixa de 15 a 19 anos. AMAZARRAY ET AL (1998) sugerem que o período da adolescência está relacionado diretamente à uma dependência social e econômica total da família. CAMPOS (2002) caracteriza a adolescência em termos de processo psicológicos, que envolvem as reações do jovem às suas mudanças físicas e à resolução razoável de sua 
identidade pessoal, que para a maioria das pessoas ocorrem entre 11 e 21 anos.

No presente artigo, optou-se pela definição proposta pelo ECA (2002, p. 11)

"art. $2^{\circ}$.. Considera-se criança, para os fins da Lei, a pessoa até doze anos de idade incompletos, e adolescente aquela entre doze e dezoito anos de idade.

Parágrafo único : nos caos expressos em Lei, aplica-se excepcionalmente este Estatuto às pessoas entre dezoito e vinte e um anos de idade " (Lei n. 8069, de 13 de julho de 1990).

A escolha por esta definição justifica-se pela importância social e política do ECA, principalmente no que se refere à proteção integral da criança e do adolescente. Além disso, MEDEIROS (1998) observa que existem alterações do curso do desenvolvimento de crianças e adolescentes de rua, refletida por um amadurecimento precoce ou por regressões e fixações em etapas determinadas da infância. Assim sendo, a caracterização da adolescência de acordo com critérios puramente psicológicos, sócio-econômicos ou outros não se mostra adequada aos propósitos deste estudo.

Independentemente da determinação da faixa etária, a adolescência é caracterizada por mudanças físicas, mentais e emocionais. Para COSTA (2001) o adolescente é um sujeito em vias de construção de sua identidade pessoal, sexual e afetiva. No que se refere à adolescentes em situação de rua, esta construção encontra-se permeada por fatores relacionados à miséria (precárias condições de habitação, educação, saúde, alimentação, etc ) e à modalidades de violência e agressão.

É nesse contexto que, segundo MEDEIROS et al (2001, pg. 37), que o adolescente "descobre a sexualidade a partir da referência de colegas que estão nas ruas há mais tempo, assim como de experiências impostas por outros adolescentes ou adultos". Em um estudo realizado na cidade de Goiânia, MEDEIROS et al (2001) pontuam que para o grupo de adolescentes em situação de rua abordados pela pesquisa, a prática do sexo seguro não faz parte de seu cotidiano. Em conseqüência, a adolescente está susceptível à transmissão/contaminação por doenças sexualmente transmissíveis e à gravidez.

\section{Gravidez e Maternidade na Adolescência: diferentes significações}

Tradicionalmente, a gravidez e maternidade na adolescência têm sido, tratadas como problemas de saúde pública no Brasil, sendo caracterizadas como situações associadas à riscos pessoais e sociais para o desenvolvimento da adolescente e de seu filho (SANTOS \& SCHOR, 2003; AMAZARRAY et al, 1998; COSTA et al, 2001; PONTE JÚNIOR \& XIMENES NETO, 2004). No entanto, cada vez mais estudos que consideram a percepção da adolescente sobre a experiência da maternidade não revelam essa unanimidade do caráter negativo da gravidez na adolescência, principalmente no que se refere à adolescentes em situação de risco social e pessoal.
De acordo com PANTOJA (2003), o fenômeno da gravidez na adolescência não se caracteriza como um fato recente no Brasil. No entanto, segundo FÁVERO \& MELLO(1997) é após a década de 60 que se nota um maior interesse no estudo deste fenômeno. Esse interesse coincide com um contexto histórico de discussões e mudanças nos valores referentes aos conceitos de gênero e sexo.

A partir deste momento, a gravidez na adolescência passa a ser considerada "um problema social", sustentada por um discurso médico que a caracterizou como quadro de gravidade e risco (PANTOJA, 2003). De acordo com PORTO \& LUZ (2002, P. 385), o Ministério da Saúde considera "a gravidez na adolescência, de alto risco, que podem ser de natureza diversa, tais como clínicas, biológicas, comportamentais, relacionadas á assistência à saúde, sócio-culturais, econômicas e ambientais".

PANTOJA (2003) e PINHEIRO (2000), no entanto discutem baseados em diversos estudos, os riscos freqüentemente associados à gravidez na adolescência. Os pesquisadores questionam se a prática do aborto em adolescentes apresenta uma maior ocorrência quando comparadas com mulheres adultas. Um outro aspecto, diz respeito aos problemas de saúde (no parto, peso ao nascer, prematuridade) que podem ser enfrentados pelas adolescentes grávidas. PINHEIRO (2000) coloca que os riscos de saúde aos quais as adolescentes estão sujeitas dizem respeito mais à situação de precariedade sócioeconômica que podem ser vivenciadas mulheres de qualquer faixa etária do que às características maturacionais da adolescente (exceto no caso de meninas abaixo de 14 anos).

Do ponto de vista da psicologia, PANTOJA (2003) discute o risco psicossocial , que seria justificado por uma imaturidade psicológica das jovens. A questão da "precocidade" da gravidez traz em seu bojo, segundo CARDOSO \& DURAND (2001) uma conotação negativa e indesejada da sexualidade precoce, assumindo um discurso "que vai da condenação ao apelo moral aos adolescentes " (BOZON apud PANTOJA, 2003, p. s336). O risco social , discurso normativo predominante na saúde pública, traz implícito uma interpretação de natureza causa de que "a gravidez resultaria da pobreza, da precaridade e da falta de acesso aos serviços de saúde, portanto é considerada como um reforço à pobreza e marginalidade. Uma vez indesejada, a gravidez acarretaria prejuízos para as adolescentes como abandono dos estudos, dificuldade em encontrar emprego." (PANTOJA, 2003, p. 336)

Diversos autores (PANTOJA, 2003; FÁVERO \& MELLO, 1997; OLIVEIRA, 1999) questionam essa visão reducionista do fenômeno, salientando que embora a gravidez na adolescência encontre-se com freqüência relacionada à um contexto de desvantagem social das garotas, é preciso considerar que sua ocorrência já se dá em um contexto de oportunidades restritas, poucas opções de vida e marcado por interrupções na trajetória escolar (PANTOJA, 2003).

Neste contexto, OLIVEIRA (1999), PANTOJA (2003), WAGNER et al (1998), COMMITTEE ON 
ADOLESCENCE AND C OMMITTE ON EARLY CHILDHOOD, ADOPTION AND DEPENDENT CARE (2001) CARDOSO \& DURAN (2001), pontuam que a maternidade pode assumir um caráter de centralidade na vida das adolescentes.

A maternidade, de acordo com SZAPIRO (2002), não pode ser reduzida aos aspectos biológicos, pois "do ponto de vista da cultura humana, não existe fato biológico em si, o que existe são, portanto, discursos próprios a cada cultura que constituem os fatos biológicos" (SZAPIRO, 2002). Entendida dessa forma, a maternidade passa a ser vista enquanto fenômeno social e consequentemente como uma construção sócio-histórica (KIMURA, 1997). De acordo com KIMURA (1997): No caso da mulher, quando vivencia o processo da gravidez, ela se identifica com uma identidade pressuposta de mãe, o quê posteriormente como representação será interiorizado e objetivado socialmente (p. 341)

Considerando estes aspectos, OLIVEIRA (2002), sugere que para adolescentes pobres, provenientes em geral de núcleos familiares matriarcais, pouco provedoras de cuidado, proteção e carinho, observa-se uma maior atração (consciente ou inconsciente) pela gravidez. Para estas adolescentes, a maternidade não era significada como algo precoce, mas sim como mais uma etapa natural do processo de desenvolvimento, sendo este aspecto também observado por CARDOSO \& DURAN (2001). De acordo com OLIVEIRA (1999) uma vez que o social provê pouco alimento psíquico, as respostas da "natureza" podem ser fontes procuradas até como defesas, perante condições de esvaziamento .

Um outro aspecto relacionado à maternidade de adolescentes em situação de risco social e pessoal, diz respeito a maternidade enquanto forma de ascensão social como um "passaporte " para a vida adulta, e como reforço pra ser alguém na vida com vistas a garantir a estima de outras pessoas e um futuro melhor para e através do filho (FAVÉRO, 1997; PANTOJA, 2003).

Ainda em relação à adolescentes em situação de risco social e pessoal, WAGNER et al (1998) sugerem que a gravidez age como um fator de proteção para a depressão em adolescentes com características pessimistas, pontuando que adolescentes emocionalmente privadas, tem descrito sentimentos de importância, de serem especiais, durante a gravidez. Em outro estudo, o Comitte on Adolescence and Comitte on Early Childhood (2001), identificou a gravidez na adolescência à diminuição do uso de álcool,cigarro, maconha e crack durante a gestação, sendo esta diminuição constatada até 6 meses pós parto, no caso de cigarro e álcool. Estes achados também são sugeridos por OLIVEIRA (2002),que em seu estudo sobre gravidez e maternidade de adolescentes de periferias sociais e ambientais, observou que pontua o cuidado dos filhos (menores que 4 anos ) aparentemente trazia benefícios psicossociais, especialmente relacionados à convivência , administração e escape dos riscos graves do mundo do tráfico, do abuso de drogas e da criminalidade.
Especificamente, no caso de adolescentes mães em situação de rua, não foram encontrados estudos no contexto brasileiro. No entanto, de acordo com PASSOS (2001) " em que pese o afastamento da casa as referências socializadas na família... sobrevivem em realidade das experiências de rua ( $p$. 674 )" , sendo importante considerar que todos os aspectos discutidos anteriormente fazem parte do cotidiano de milhares de adolescentes em situação de risco social e pessoal, sendo a opção de ir para a rua relacionada, principalmente, ao sucesso ou fracasso da integração familiar. Neste contexto, a gravidez encontra sua razão de ser em uma estratégia de afirmação e identidade de adolescentes, em famílias de pouca integração. Não tendo outra expectativa de vida, estas adolescentes fazem do ser mãe seu projeto de vida .

\section{CONSIDERAÇÕES FINAIS}

Por meio desta revisão não pretendemos negar ou negligenciar $o$ impacto $e$ as conseqüências negativas da vivência da gravidez na adolescência, período caracterizado por mudanças extremamente importantes para o desenvolvimento da mulher. No entanto, como nos confirma MOREIRA (2003) existe uma contradição entre o discurso sobre a gravidez na adolescência que a qualifica como um problema, e a prática cotidiana das jovens que a experimentam, principalmente no que se refere à adolescentes em situação de risco social e pessoal.

Para as adolescentes, especialmente aquelas em situação de risco social e pessoal, a maternidade pode adquirir um caráter de centralidade em suas vidas, sendo um importante fator na sua constituição pessoal e social, pois traz interferências sobre novas formas de relacionamentos e reconhecimentos sociais e de atuação em seu cotidiano. Tal caráter também é percebido no momento em que buscam alternativas de vida para si e para seu filho, que propicia um momento importante para o desenvolvimento ações de saúde e sociais que visem oferecer assistência adequada e efetiva para essa população. Nesse sentido concordamos com SARTORI \& VAN DER SAND (2004) quando apresentam a estratégia grupal na assistência à gestante como um importante espaço de trocas de experiências e criação e/ou fortalecimento de vínculos entre as participantes, tornando-se assim uma importante ferramenta como forma de assistir de modo integral às pessoas, no caso as adolescentes.

Assim sendo, entendendo a maternidade enquanto fenômeno socialmente constituído, buscouse neste artigo polemizar as discussões sobre aspectos tradicionalmente abordados na literatura, bem como instigar a elaboração de pesquisas que abordem a maternidade na adolescência em um eixo sócio-cultural, fornecendo desta forma subsídios para a elaboração de políticas sócias e de saúde adequadas a essa população .

REFERÊNCIAS BIBLIOGRÁFICAS: 
AMAZARRAY, M. R.; MACHADO, P. S., OLIVEIRA, V. Z. GOMES, W. B. A experiência de assumir a gestação na adolescência: um estudo fenomenológico. Psicologia: reflexão e crítica, v. 11, n. 3, p. 431-440, 1998.

BRASIL . Estatuto da Criança e do Adolescente Lei n. 8.069, de 13 de julho de 1990. Secretaria de Estado dos Direitos Humanos - Departamento da Criança e do Adolescente. Brasília, 2002.

CARDOSO, E. B.; DURAND, V. Gravidez na Adolescência . In: GICO, V.; SPINELLI, A; VICENTE, P. As Ciências Socias: Desafios do Milênio. Natal: EDUFRN, 2001. p. 649-667

CARVALHO, M. C. B.; GUARÁ, I. M. F. R. A família: um sujeito pouco refletido no movimento de luta pelos direitos da criança e do adolescente. Rev. Brás. Cresc. Desenv. Hum., v. 4,. n. 1, 1994. p. 45-48, 1994.

COMMITTEE ON ADOLESCENCE AND COMMITTE ON EARLY CHILDHOOD, ADOPTION AND DEPENDENT CARE Care of Adolescent Parents and their children. Pediatrics, v. 107, n. 2, p.429-434, 2001. COSTA, S. M. S. Vivendo com AIDS e enfrentando a violência: experiência das adolescentes. Rio de Janeiro: ENSP/FIOCRUZ, 2000.

COSTA, L. F.; BRANCO, M. H.C., ALVES, A M. O; CRUZ, T. S. A contribuição da Terapia Ocupacional com gestantes adolescentes na maternidade Cândido Mariano em Campo Grande. Anais do VII Congresso Brasileiro de Terapia Ocupacional. Porto Alegre, 2001. FAVERO, M H.; MELLO, R M Adolescência, Maternidade e vida escolar: a difícil conciliação de papéis. Psicol. Teor. Pesqu, v. 13, n. 1, p. 131-136, 1997.

GALHEIGO, S. M., VILAS BOAS, F. D. CLARETO, L.F. ALLEGRETTI, M. M.; SILVA, S. M. Pensando a rede de proteção especial: um estudo sobre a atenção à criança em situação de risco pessoal e social na cidade de Campinas. Anais do VII Congresso Brasileiro de Terapia Ocupacional. Porto Alegre, 2001. KIMURA, A F A construção da personagem mãe: construções teóricas sobre identidade e papel materno. Revista da Escola de Enfermagem da USP, v. 31, n. 2, p. 339-343, 1997.

LESCHER, A. D.; GRAJCER, B.; BEDOIN, G.; AZEVEDO, L. M.; SILVA, L. N.; PERNANBUCO, M. C. A.; JUNIOR, N. C. Crianças em situação de risco social: limites e necessidades da atuação do profissional de saúde. Disponível em: http://www.projetoquixote.epm.br/publicacao.pdf.

Acesso em 12 de maio de 2005.

MEDEIROS, M.; FERRIANI, M. G. C. Programas de Atenção às crianças e aos adolescentes em situação de rua: percepções de seus coordenadores. Revista Brasileira de Crescimento e Desenvolvimento Humano, V. 5, n. 1/2, p. 48-49, 1995.

MEDEIROS, M; FERRIANI, M G C; MUNARI, D.B.; GOMES, R. A sexualidade para o adolescente em situação de rua em Goiânia. Revista Latino Americana de Enfermagem, v. 9, n. 2, p. 35-41, 2001.

MEDEIROS, M; FERRIANI, M G C; GOMES, R; MUNARI, D B O significado de casa e rua para meninos com experiência de vida nas ruas: em busca de uma compreensão sobre as implicações para a saúde. Revista Brasileira de Crescimento e Desenvolvimento Humano, v. 12, n. 2, p. 1-12, 2002. MELLO, S. L. Famílias das Classes Populares: tradição e mudança. Revista Brasileira de Crescimento e Desenvolvimento Humano. Vol 4, n. 1, 1994, pgs. 21-27

MOREIRA, M. I. C. Laços familiares e laços geracionais: uma reflexão sobre a gravidez entre mulheres adolescentes. In: SOUSA, S. M. Infância e Adolescência: múltiplos olhares. Goiânia, Editora UCG, 2003, p. 113-144

OLIVEIRA, $\mathrm{N} \mathrm{R}$ Perfis de grávidas e mães adolescentes: estudo psicossocial de adolescentes usuárias de um serviço público de pré-natal e maternidade. São Paulo: (tese de doutorado) Instituto de Psicologia USP, 1999

OLIVEIRA, N R Gravidez e Maternidade de adolescentes de periferias sociais e urbanas: reflexões à luz da Psicologia Ambiental. Relatório de Pesquisa (pós doutorado) Departamento Materno infantil, Faculdade de Saúde Pública, USP, 2002

PANTOJA, A L N "Ser alguém na vida: uma análise sócio-antropológica da gravidez/maternidade na adolescência em Belém do Pará, Brasil. Cad. Saúde Pública, Rio de Janeiro, 19 (supl. 2) , 2003, S335-S343 PASSOS, K R C Memória e Identidade Social de Meninas(os) de rua em Belém .In: GICO, V.; SPINELLI, A ; VICENTE, P. As Ciências Socias: Desafios do Milênio. Natal: EDUFRN, 2001. p. 668-679 PINHEIRO, V S Repensando a maternidade na adolescência. Estudos de Psicologia, 5 (1), p. 243-251, 2000

PONTE JUNIOR, G. M.; XIMENES NETO, F. R. G. Gravidez na adolescência no município de Santana do Acaraú - Ceará - Brasil: uma análise das causas e riscos. Revista Eletrônica de Enfermagem, v. 06, n. 01, $2004 . \quad$ Disponível em http://www.fen.ufg.br/revista/revista6 1/f3 gravidez.ht ml. Acesso em 29 Jul 2004.

PORTO, J R R ; LUZ, A M H Percepções da adolescente sobre a maternidade. Revista Brasileira de Enfermagem, 55(4), 384-391, jul-ago.2002

RIZZINI, I.; SILVA, N. C. R. Direitos humanos e direitos da criança e do adolescente: reflexões sobre desigualdades sociais e a questão dos "meninos de rua". In: SOUSA, S. M. (org). Infância e Adolescência: múltiplos olhares. .Goiânia: Editora da UCG, 2002, pg 99-110.

SANTOS, S. R. ; SCHOR, M. H. C. Vivências da maternidade na adolescência precoce. Revista de Saúde Pública. Vol 37, n. 1, fev. 2003 pgs 15-23

SARTI, C. A A continuidade entre casa e rua no mundo da criança pobre. Revista Brasileira de Crescimento e Desenvolvimento Humano.vol 5, n. 1/2, 1995, pg. 39-47

SARTORI, G. S.; VAN DER SAND, I. C. P. Grupo de gestantes: espaço de conhecimentos, de trocas e de vínculos entre os participantes. Revista Eletrônica de Enfermagem, v. 06, n. 02, 2004. Disponível em http://www.fen.ufg.br/revista/revista6 2/gestantes.html. Acesso em 29 Jul 2004.

SZAPIRO, A M ; FÉRES-CARNEIRO, T. Construções do feminino pós anos sessenta: o caso da maternidade 
como produção independente. Psicol. Reflex. Crit. 15(1), 179-188, 2002

WAGNER, K D ; BERENSON, A , HARDING, O; JOINER, T. Attritional style and depression in pregnant teenagers . AM J Psychiatry, set, p. 155-159, 1998

Texto recebido em 09/08/2004

Publicação aprovada em 10/12/2004 